\title{
Flexible Bio-tensegrity Manipulator with Multi-degree of Freedom and Variable Structure
}

\author{
Dunwen Wei ${ }^{{ }^{*}}$ (D), Tao Gao ${ }^{1}$, Xiaojuan $\mathrm{Mo}^{2}$, Ruru Xi ${ }^{3}$ and Cong Zhou ${ }^{1}$
}

\begin{abstract}
Conventional manipulators with rigid structures and stiffness actuators have poor flexibility, limited obstacle avoidance capability, and constrained workspace. Some developed flexible or soft manipulators in recent years have the characteristics of infinite degrees of freedom, high flexibility, environmental adaptability, and extended manipulation capability. However, these existing manipulators still cannot achieve the shrinking motion and independent control of specified segments like the animals, which hinders their applications. In this paper, a flexible bio-tensegrity manipulator, inspired by the longitudinal and transversal muscles of octopus tentacles, was proposed to mimic the shrinking behavior and achieve the variable motion patterns of each segment. Such proposed manipulator uses the elastic spring as the backbone, which is driven by four cables and has one variable structure mechanism in each segment to achieve the independent control of each segment. The variable structure mechanism innovatively contains seven lock-release states to independently control the bending and shrinking motion of each segment. After the kinematic modeling and analysis, one prototype of such bionic flexible manipulator was built and the open-loop control method was proposed. Some proof-of-concept experiments, including the shrinking motion, bending motion, and variable structure motion, were carried out by controlling the length of four cables and changing the lock-release states of the variable structure mechanism, which validate the feasibility and validity of our proposed prototype. Meanwhile, the experimental results show the flexible manipulator can accomplish the bending and shrinking motion with the relative error less than $6.8 \%$ through the simple independent control of each segment using the variable structure mechanism. This proposed manipulator has the features of controllable degree-of-freedom in each segment, which extend their environmental adaptability, and manipulation capability.
\end{abstract}

Keywords: Bio-tensegrity, Cable-driven actuation, Multi-degree-of-freedom (Multi-DoF), Flexible manipulator, Variable structure mechanism

\section{Introduction}

The technologies about the mechanical design and motion control of traditional robots are relatively mature. However, traditional robots for heavy industrial applications usually utilize rigid materials, inelastic articulation components, and stiffness actuators, which have severe limitations in other circumstances, such as collaboration

\footnotetext{
*Correspondence: weidunwen@uestc.edu.cn

${ }^{1}$ School of Mechanical and Electrical Engineering, University of Electronic

Science and Technology of China, Chengdu 611731, China

Full list of author information is available at the end of the article
}

and manipulation in confined space [1]. In the complex and unstructured environment, traditional robots are limited by their own rigid structures, which also restrict their obstacle avoidance and manipulation capabilities [2]. Those traditional robots have relatively poor flexibility and adaptability and therefore cannot efficiently complete some tasks in a safe manner, especially involving the physical human-robot interaction. In contrast, looking back on the animals in the biological world, they are characterized by soft and compliant substance $[3,4]$, such as their skin, limbs, muscles, even including muscular hydrostatic skeletons [5]. In order to deal 
with the challenges of traditional robots, some flexible or soft robots have been developed with the characteristics of infinite degrees of freedom (DoF), high flexibility, environmental adaptability, and extended manipulation capability in recent years. There are three roadmaps to achieve the compliance characterized by the nature animals, including soft robot with intrinsic actuation [6], continuum robots with extrinsic actuation, and traditional robots with elastic/compliant actuation [7].

Soft robots are a class of robots using soft materials including gases, liquids, polymers, foams, gels, colloids, granular materials, as well as most soft biological materials [8-11]. Inherent compliance of gas and liquid make soft pneumatic actuator and hydraulic actuator as good candidates for many challenging tasks [12-14]. Some work pointed out that soft pneumatic actuator have some considerable characteristics, such as multiple degree of freedom (Multi-DoF) actuation [12], high force, and reliability [13]. Shiva et al. [15] proposed a pneumatically continuum manipulator with embedded chambers in the soft silicone structure. However, the overall size and weight of peripheral device such as compressor unit, on-board electronics, confines the possibility of using these actuators. Sometimes, the term of soft robot also can be especially described a class of robots using some advanced functional materials with intrinsic actuation as the soft-body material, such as shape memory polymer (SMP) [16], shape memory alloy (SMA) [17-19], electroactive polymer (EAP) [20,21], and so on, therefore soft robots are featured with physical self-driven characteristics $[16,22]$. Soft material have great prospects in the small-scale robots because of their inherent advantages, such as lightweight, compliance, high strain with continuum deformation. However, full soft material robots are usually not able to achieve some functional requirements including large force, fast motion and controllability from the current research status.

Continuum robot is used here to especially refer to an actuatable structure with extrinsic actuation. Continuum robot usually have discrete structure, multi-backbone, and driven by tendon/cables [23]. Many continuum manipulators are usually proposed. An elephant's trunk robot described in Ref. [3], with very few driving constraints, consists of a series of novel underactuated linkage units and a single motor to drive, which can achieve stable grasping of objects with different shapes and sizes. A multi-section soft manipulator dynamics was presented based on a discrete Cosserat approach [24]. The model can accommodate continuum robots having any number of sections with varying physical dimensions [25]. Yeshmukhametov et al. [26] presented a novel approach of kinetic, kinematic modeling and design solutions of the wire-driven universal joint backboned continuum robot. However, existing continuum robots with multiple segments require the separate power source to actuate different segments [25], therefore, the dependent control and planning process is very complicated.

Traditional robot combined with elastic/compliant actuator $[27,28]$ is another recipes to obtain structural compliance. The advantages of elastic/compliant actuator is that series elastic actuators [27] and variable stiffness actuators [28] combine the elastic element or compliant mechanism with conventional stiffness actuators to regulate energy transmission [29], to improve energy efficiency, and to achieve safety physical interaction. Ham and Sugar et al. surveyed the various elastic actuators with passive adjustable compliance and controllable stiffness for robotic applications [28]. However, the typical elastic/compliant actuators comply with compliance only along their axes of rotation. Structural compliance is hard to achieve motion control of Multi-DoF. Therefore, this drawback restricts the range of motion space.

Comprehensive research on soft and continuum robots has accomplished significant progress in recent years, but the following deficiencies generally remain: a) Environmental adaptability in extreme and unstructured environment. b) Complex control of multiple segments. Existing continuum robots require the separate power source to actuate different segments [25].

Inspired by the biological feature and mechanism of the octopus tentacles, a kind of novel flexible manipulator based on the bio-tensegrity structure is proposed in this paper. The underlying beauty of the proposed flexible bio-tensegrity manipulator is manifold. Firstly, the conventional materials, including springs and cables, are used in the proposed method to constitute a flexible bio-tensegrity manipulator, enabling to mimic the behaviors of transversal and longitudinal muscles. This tensegrity mechanism is not only lighter and easier to handle for actuation [30], but also has the main advantage of high load capacity. Secondly, each segment with Multi-DoF can be independently controlled to achieve the shrinking and bending motions through the variable structure mechanism, while there is no need for a separate actuation source. The variable structure mechanism innovatively contains seven lock-release states to control the variable motion pattern of the flexible bio-tensegrity manipulator. This flexible bio-tensegrity manipulator not only has continuum deformation and Multi-DOF as soft materials but also has better stability against various decays and is easier for modeling and control.

The main contents of this paper include the following aspects. Firstly, inspired by the biological principle of the octopus tentacles, the novel manipulator based on the bio-tensegrity structure is proposed in Section 2. Then, 
the simplified modeling and kinematic analysis of the flexible manipulators are carried out in Section 3. After that, one manipulator prototype is constructed according to the above design principles. After integrating the mechanism and controlling modules, the performance and effectiveness of the bionic flexible tensegrity manipulator are validated by some proof-of-concept experiments on this platform in Section 4. Finally, Section 5 discusses the conclusions and future work to be conducted.

\section{Bionic Design Method and Mechanism}

\subsection{Inspiration of the Octopus Tentacles}

Lives in nature have evolved over hundreds of millions of years, and the mechanisms of each species are complex and perfect [4]. Inspired by biological specimens, such as elephant trunks [3], earthworm [31], and snakes [32], various continuum robots were proposed and built to traverse confined spaces, manipulate objects in complex environments. Through the study of mollusk octopus in the animal kingdom, it is found that the octopus can not only rely on its soft body to adapt to various obstacles but also can freely bend, twist and shrink the body's shape of different segments to achieve movements such as peristalsis and fluctuation. As shown in Figure 1, the octopus is a typical muscular hydrostatic skeletal structure (Additional file 1). The tentacles of octopus are capable of actuating the different segments of muscles, in such a way, that it can control its body length by shrinking as the A region shown in Figure 1(a) and bend the specified segment as the B region in Figure 1(a) of its arm, enabling the animal to complete extremely complex movements, such as obstacle avoidance, catching and gripping fish or even walking across the seabed. Figure 1(b) is an anatomical view of the octopus tentacles [33], where $\boldsymbol{T}$ represents the transversal muscle; $\boldsymbol{L}$ represents the longitudinal muscle; $\boldsymbol{O}$ represents the external oblique muscle, and $N$ denotes the nerve cord. At the same time, the soft has the following features: a) the soft tentacle with an infinite degree of freedom to enhance environment adaptability; b) independently controlling the motion patterns of each segment to bend and shrink.

The tentacles have four longitudinal muscles and one transversal muscle to provide the actuation power. We can use four flexible or soft actuation elements and one center actuation element to mimic the motion of the octopus tentacle. The technologies of the soft-material robot just satisfy the requirements of achieving those features. Nevertheless, the full soft materials with inherent compliance are by no means fitted for building the large force and fast motion applications from the current research status. Meanwhile, soft robots aren't able to achieve functional requirements of large forces and controllability.

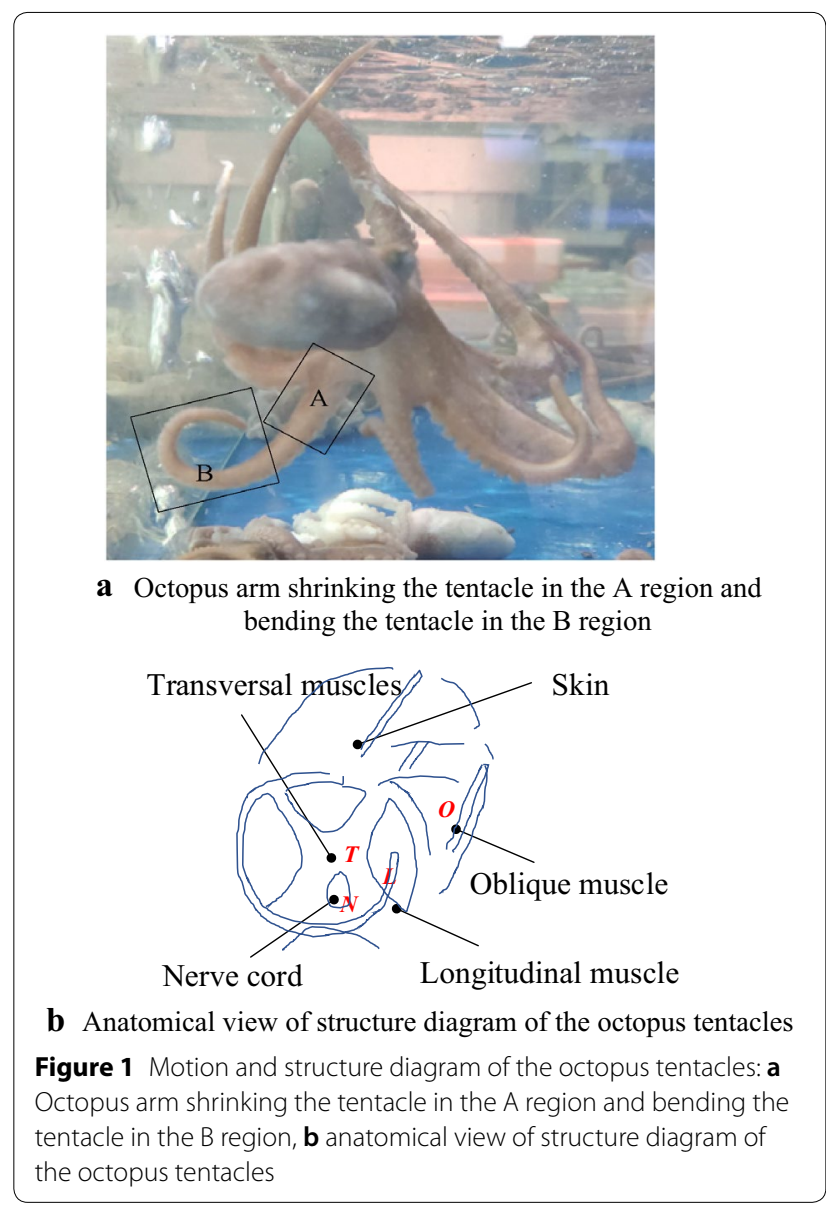

\subsection{Design of Flexible Tensegrity Manipulator}

From the conventional view of design,it is relatively hard to implement Multi-DOF and such great adaptability as the octopus. The original method to achieve space flexibility is using a parallel mechanism. The parallel mechanism is a closed-loop mechanism with two or more degrees of freedom and is driven in parallel on a moving platform, and another platform that is fixed is connected by at least two independent motion chains, as shown in Figure 2(a). Because of the rigid bracing piece and telescopic links, the motion space and flexibility are limited. The overall weight is too large, which contributes to poor safety performance.

In order to achieve the flexibility and lightweight design, super-elastic rods or strings are the extensive strategies to build the parallel continuum robots [12] Unfortunately, the super-elastic materials have no function of segmented control to achieve both bending and shrinking motion at the same time. Meanwhile, the nonlinear elasticity limits the stability and real-time control with conventional control methods. 


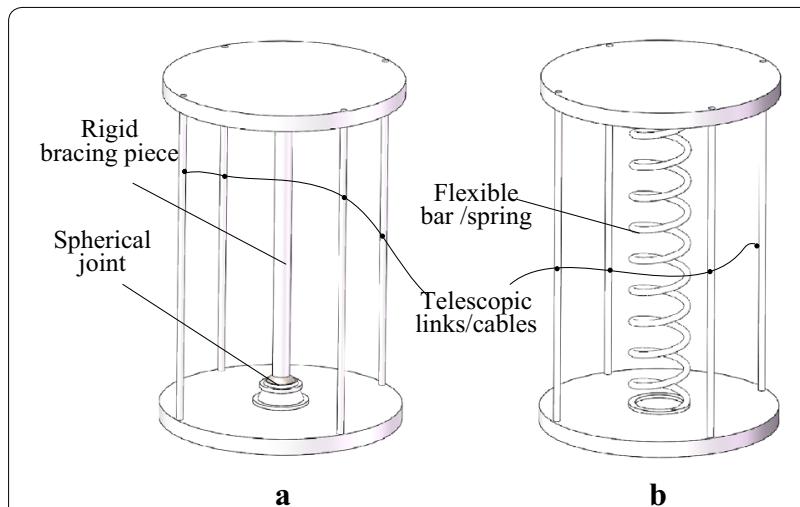

Figure $\mathbf{2}$ Various Multi-DOFs mechanisms: a 6 DOF parallel mechanism, $\mathbf{b}$ flexible 6 DOF bio-tensegrity mechanism

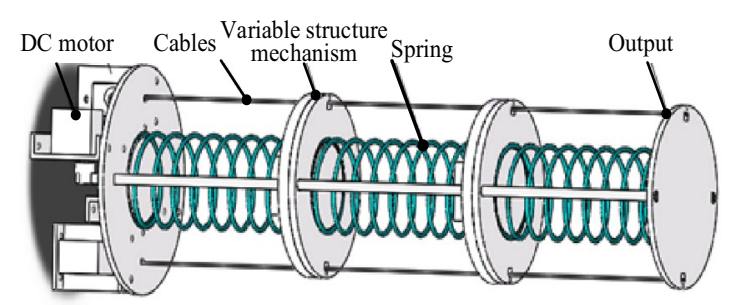

Figure 3 Flexible bio-tensegrity manipulator with three compliant mechanism segments

Therefore, we proposed the bio-tensegrity mechanism with the cable-driven and compliant element. The bio-tensegrity mechanism manipulator proposed in this paper adopts the idea of tensegrity design and the inspiration of octopus tentacles' structure. As shown in Figure 2(b), the rigid body structure in the middle is replaced by the elastic material to mimic the transverse muscle. When the mechanical arm moves to a certain position within a certain range, the elastic arm can be elasticized. The material is considered as a rigid body, at which point the position of the arm can be determined. Secondly, each segment has an independent control to achieve its bending and shrink. Therefore, the segment can achieve telescopic motion because of the existence of elastic materials. Additionally, the driving element is used as little as possible. To a certain extent, the reliability is enhanced.

The overall 3D model of the proposed bionic flexible manipulator is shown in Figure 3, which includes three segments. This 3D model contains two variable structure mechanisms, three spring elements, four cables, and four actuators. Four actuators, realized by four DC motors in our prototype, respectively drive the four cables that distribute 90 degrees around the continuous

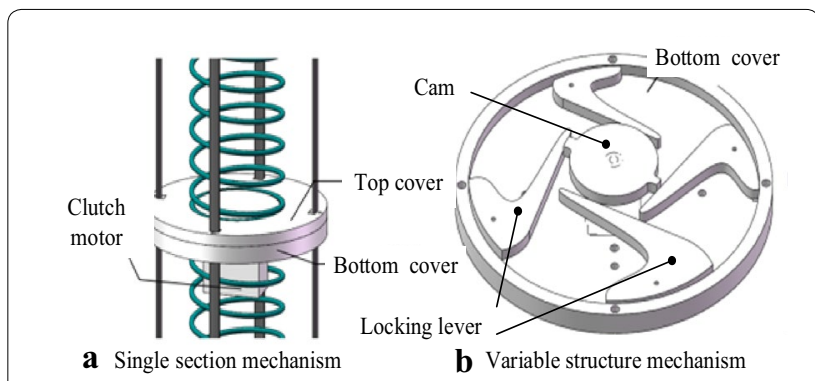

Figure 4 Segment connection device mechanism

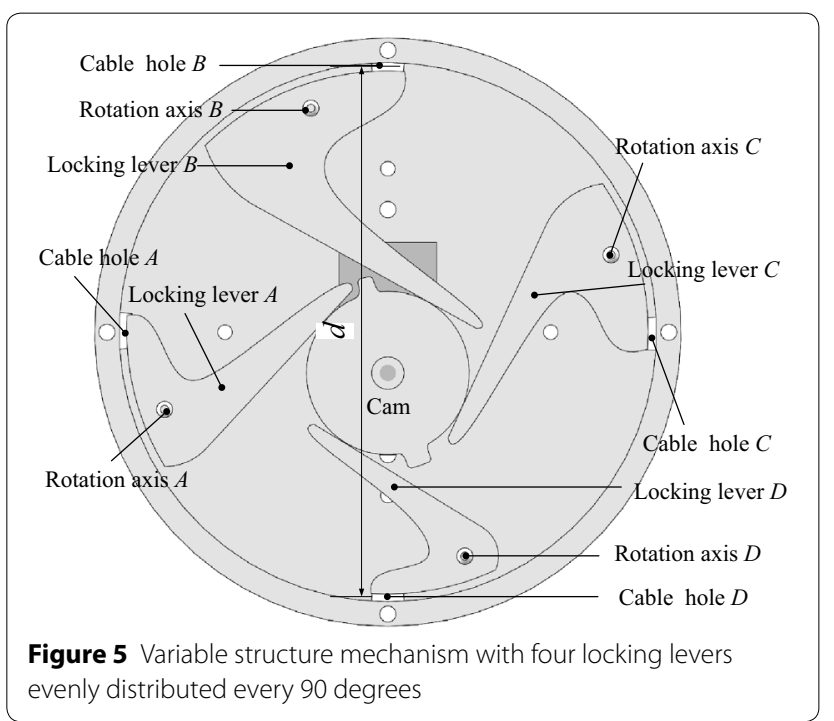

flexible manipulator. Changing the length of the cables can achieve continuous deformation in a certain direction.

The implementation of variable structure in the control of the bionic flexible manipulator is due to the presence of a built-in variable structure mechanism. The diagram of the variable structure mechanism is shown in Figure 4(a) and (b), which includes the top cover, the bottom cover, the lock motor, and the internal variable structure mechanism. The internal variable structure mechanism is the lock-release mechanism that realizes the locking or releasing state regulation of cables. The lock-release mechanism adopts the improved eccentric force pressing mechanism, which has the advantages of simple structure and convenient control. In this proposed prototype, one clutch motor can control the locking and releasing state of the four cables in different directions.

The variable structure mechanism is contained in the midst of the top cover and the bottom cover to improve reliability. The principle of the variable structure mechanism is shown in Figure 5, which comprises one cam, four locking levers, and four cable holes. The cam is 
actuated by the clutch motor and rotate to one accurate position. When the clutch motor actuates the cam to one proper position, the protruding part of the cam will push the locking lever to rotate around the rotation axis, and the locking lever will tightly press the corresponding cable through the cable hole. The cable length in this segment cannot be changed if the cable was locked through the cable hole. The variable structure mechanism can achieve seven kinds of locking states including the state in Figure 5 and states from Figure 6(a) to Figure 6(f). The corresponding states can be found in Table 1 .

Figure 6 is a schematic view showing the position of the locking device before and after the rotation, and the structure of the other three locking states Figure 6(b)(d) can be obtained in sequence. Figure 6(e) shows the simultaneous locking of cable $A$ and $C$, in which case the axial expansion and contraction of the overall mechanical device can be achieved. Figure 6(f) shows the simultaneous locking of cable $B$ and $D$, in which case the axial expansion and contraction of the overall mechanical device can be achieved.

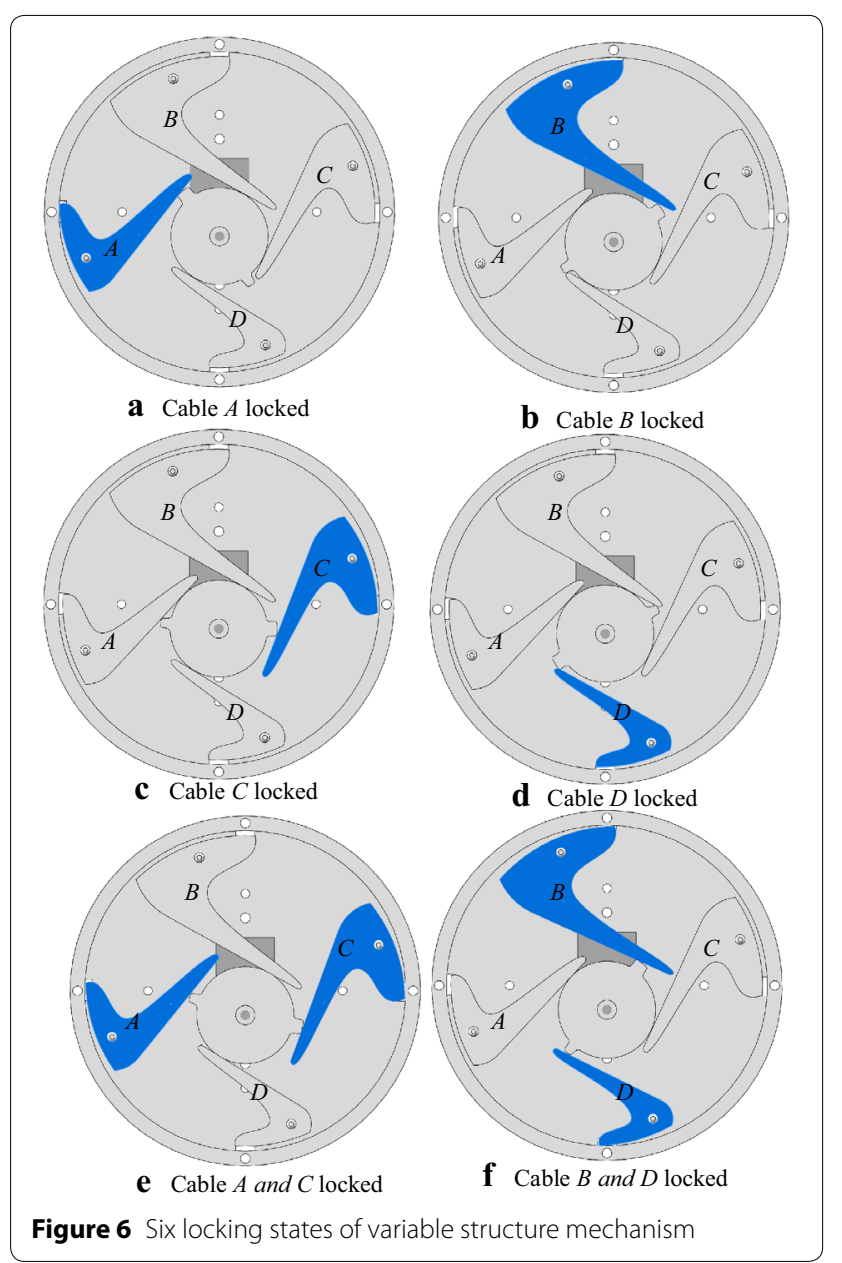

Table 1 Seven variable structure states

\begin{tabular}{lllll}
\hline States & $\begin{array}{l}\text { Cable } \\
\text { A state }\end{array}$ & $\begin{array}{l}\text { Cable B } \\
\text { state }\end{array}$ & $\begin{array}{l}\text { Cable C } \\
\text { state }\end{array}$ & Cable D state \\
\hline Figure 5 & $\mathrm{R}$ & $\mathrm{R}$ & $\mathrm{R}$ & $\mathrm{R}$ \\
Figure 6(a) & $\mathrm{L}$ & $\mathrm{R}$ & $\mathrm{R}$ & $\mathrm{R}$ \\
Figure 6(b) & $\mathrm{R}$ & $\mathrm{L}$ & $\mathrm{R}$ & $\mathrm{R}$ \\
Figure 6(c) & $\mathrm{R}$ & $\mathrm{R}$ & $\mathrm{L}$ & $\mathrm{R}$ \\
Figure 6(d) & $\mathrm{R}$ & $\mathrm{R}$ & $\mathrm{R}$ & $\mathrm{L}$ \\
Figure 6(e) & $\mathrm{L}$ & $\mathrm{R}$ & $\mathrm{L}$ & $\mathrm{R}$ \\
Figure 6(f) & $\mathrm{R}$ & $\mathrm{L}$ & $\mathrm{R}$ & $\mathrm{L}$ \\
\hline
\end{tabular}

Note: $R$ denotes the releasing state, $L$ denotes the locking state

\section{Modeling and Analysis}

In this section, the kinematics modeling of the proposed manipulator are introduced. In the relevant modeling and analysis, firstly we considered the motion of a single segment in space, then extended such method to the whole model.

\subsection{Single Segment Model}

The length of each cable determines the motion of the manipulator. The configuration of the $i$ th segment in 3D space shown in Figure 7 can be controlled by changing the length of the $L_{A i}, L_{B i}, L_{C}, L_{D i}$ cables. In order to analysis the position coordinates of each node, the global frame is fixed at the base of manipulator and denoted as $O_{0} \sim\left(x_{0}, y_{0}, z_{0}\right)$, and the local frame $i-1$ is attached at the head disk of the $i$ th segment. As shown in Figure 7, the local frame of the $i$ th segment is described as $O_{i-1} \sim\left(x_{i-1}\right.$, $\left.y_{i-1}, z_{i-1}\right)$. The axis $z_{i-1}$ is the normal of the disk $i-1$ plane. Meanwhile, the local frame $i$ is attached at the end disk of the $i$ th segment. The central angle of curve $\theta_{i}$ is the subsegment bending angle. The bending plane angle is $\phi_{i}$. The subsegment twist angle denoted as $\varepsilon_{i}$ is

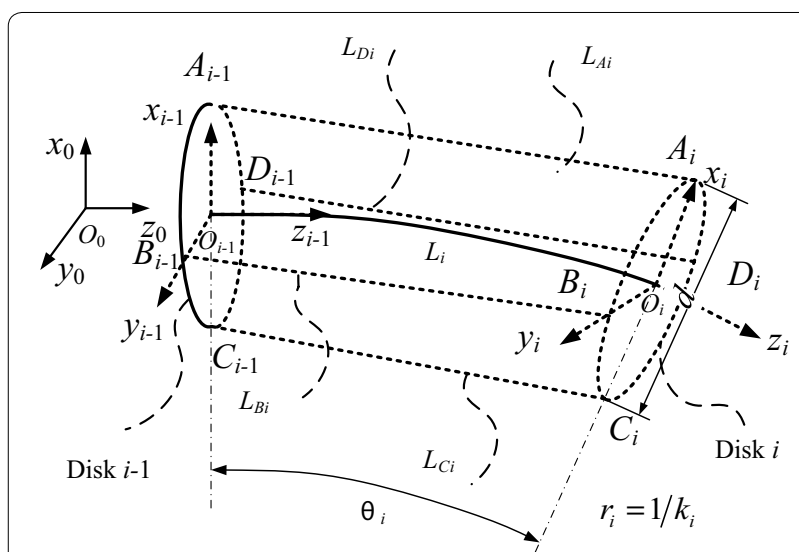

Figure 7 Position coordinates, parameters and frame definition of single segment in the workspace 
the curvature corresponding to torsion in the local $z_{i-1}$ direction. The subsegment curvature magnitude $k_{i}$. The subsegment length $l_{i}$ is the spring length of spring after deformation. $L_{i}$ is the original length of spring in the $i$ th segment.

The local rotation matrix and the center point coordinate can be expressed as follows:

$$
\begin{aligned}
& \boldsymbol{R}_{i}^{i-1}=\boldsymbol{R}_{z}\left(\varphi_{i}\right) \boldsymbol{R}_{y}\left(\theta_{i}\right) \boldsymbol{R}_{z}\left(\varepsilon_{i}-\varphi_{i}\right), \\
& \boldsymbol{P}_{i}^{i-1}=\left[\begin{array}{lll}
p_{O_{i}, x}^{i-1}, & p_{O_{i}, y}^{i-1}, & p_{O_{i}, z}^{i-1}
\end{array}\right]^{\mathrm{T}},
\end{aligned}
$$

where

$$
\begin{aligned}
& \boldsymbol{R}_{z}(\theta)= {\left[\begin{array}{lll}
\cos \theta & -\sin \theta & 0 \\
\sin \theta & \cos \theta & 0 \\
0 & 0 & 1
\end{array}\right], } \\
& \boldsymbol{R}_{y}(\theta)= {\left[\begin{array}{ccc}
\cos \theta & 0 & \sin \theta \\
0 & 1 & 0 \\
-\sin \theta & 0 & \cos \theta
\end{array}\right], } \\
& P_{O_{i}, x}^{i-1}= \cos \varphi_{i}\left(1-\cos \theta_{i}\right) / k_{i}, \\
& P_{O_{i}, y}^{i-1}= \sin \varphi_{i}\left(1-\cos \theta_{i}\right) / k_{i}, \\
& P_{O_{i}, z}^{i-1}=\sin \theta_{i} / k_{i} .
\end{aligned}
$$

The positions of cable holes can be expressed in the frame $O_{i-1} \sim\left(x_{i-1}, y_{i-1}, z_{i-1}\right)$ as follows:

$$
\begin{aligned}
& \boldsymbol{P}_{A_{i-1}}^{i-1}=[d / 2,0,0]^{\mathrm{T}}, \boldsymbol{P}_{B_{i-1}}^{i-1}=[0, d / 2,0]^{\mathrm{T}}, \\
& \boldsymbol{P}_{C_{i-1}}^{i-1}=[-d / 2,0,0]^{\mathrm{T}}, \boldsymbol{P}_{D_{i-1}}^{i-1}=[0,-d / 2,0]^{\mathrm{T}}, \\
& \boldsymbol{P}_{A_{i}}^{i-1}=\boldsymbol{P}_{i}^{i-1}+\boldsymbol{R}_{i}^{i-1}[d / 2,0,0]^{\mathrm{T}}, \\
& \boldsymbol{P}_{B_{i}}^{i-1}=\boldsymbol{P}_{i}^{i-1}+\boldsymbol{R}_{i}^{i-1}[0, d / 2,0]^{\mathrm{T}}, \\
& \boldsymbol{P}_{C_{i}}^{i-1}=\boldsymbol{P}_{i}^{i-1}+\boldsymbol{R}_{i}^{i-1}[-d / 2,0,0]^{\mathrm{T}}, \\
& \boldsymbol{P}_{D_{i}}^{i-1}=\boldsymbol{P}_{i}^{i-1}+\boldsymbol{R}_{i}^{i-1}[0,-d / 2,0]^{\mathrm{T}} .
\end{aligned}
$$

As shown in Figure 7, the cable $A$ pass through the cable hole $A_{i-1}$ in the Disk $i-1$ and the cable hole $A_{i}$ in the Disk $i$. Similarly, the cable $B, C, D$ cable pass through $B_{i-1} B_{\mathrm{i}}, C_{i-1} C_{\mathrm{i}}, D_{i-1} D_{i}$. Therefore, the nominal length of cable $A, B, C$, and $D$ in the $i$ th segment can be expressed as the distance of two corresponding holes, shown below:

$$
\begin{aligned}
L_{A_{i}} & =\left\|\boldsymbol{P}_{A_{i}}^{i-1}-\boldsymbol{P}_{A_{i-1}}^{i-1}\right\|_{2}, \\
L_{B_{i}} & =\left\|\boldsymbol{P}_{B_{i}}^{i-1}-\boldsymbol{P}_{B_{i-1}}^{i-1}\right\|_{2}, \\
L_{C_{i}} & =\left\|\boldsymbol{P}_{C_{i}}^{i-1}-\boldsymbol{P}_{C_{i-1}}^{i-1}\right\|_{2}, \\
L_{D_{i}} & =\left\|\boldsymbol{P}_{D_{i}}^{i-1}-\boldsymbol{P}_{D_{i-1}}^{i-1}\right\|_{2}
\end{aligned}
$$

Figure 8 describes the special case of the $i$ th segment after deformation when the $\varphi_{i}=0$ and $\varepsilon_{i}=0$. In this case, the cable $A$ is contracted and this segment bends by $\theta_{i}$ around $y_{i-1}$. The cable $B$ and cable $D$ overlap after projection to the plane $O_{i-1} x_{i-1}, z_{i-1}$. The relationship between the cable length and bending angle $\theta_{i}$ can be expressed as follows:

$$
\cos \theta_{i}=\frac{2 d^{2}-\left(L_{C i}-L_{A i}\right)^{2}}{2 d^{2}} .
$$

\subsection{N Segments Model}

The $\mathrm{N}$-segment model can use the recursive method. The position coordinates at the end of each segment model is determined by the length of the four cables, therefore, there are totally $4 N$ parameters determining the position and configuration of the manipulator. The global orientation $\boldsymbol{R}_{i}$ of disk $i$ can be expressed as follows:

$$
\boldsymbol{R}_{i}= \begin{cases}\boldsymbol{R}_{i}^{i-1}, & i=1, \\ \boldsymbol{R}_{i-1} \boldsymbol{R}_{i}^{i-1}, & 2<i<N .\end{cases}
$$

The global position $\boldsymbol{P}_{i}$ of disk $i$ can be expressed as:

$$
\boldsymbol{P}_{i}= \begin{cases}\boldsymbol{P}_{i}^{i-1}, & i=1, \\ \boldsymbol{P}_{i-1}+\boldsymbol{R}_{i-1} \boldsymbol{P}_{i}^{i-1}, & 2<i<N .\end{cases}
$$

The total length of each cable can be expressed as:

$$
\begin{aligned}
L_{A} & =\sum_{i=1}^{i=N} L_{A i}, \\
L_{B} & =\sum_{i=1}^{i=N} L_{B i}, \\
L_{C} & =\sum_{i=1}^{i=N} L_{C i}, \\
L_{D} & =\sum_{i=1}^{i=N} L_{D i} .
\end{aligned}
$$

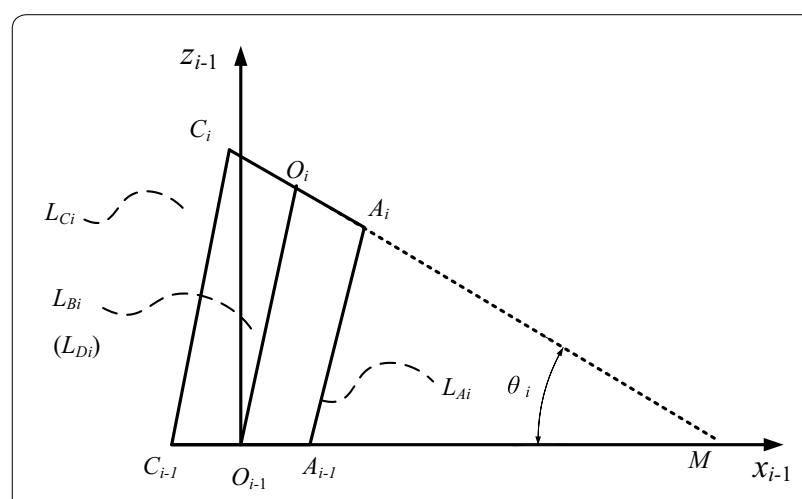

Figure 8 The case of the ith segment if $\varphi_{i}=0$ and $\varepsilon_{i}=0$ 
Figure 9 depicts the projection graph obtained by $N$-segment model intercepting the projection of the end three sections of the model when the $\varphi_{i}=0$ and $\varepsilon_{i}=0$. The position coordinates of $O_{n}$ and configuration of the full manipulator are obtained by the recursive method.

\section{Experiments of Principle Prototype}

\subsection{Prototype and Control System}

In order to verify the performances of the proposed flexible bio-tensegrity manipulator, one primary prototype shown in Figure 10 was built according to the 3D model described in Figure 3. Considering the model structure and the force status of each component, the ABS material was selected as $3 \mathrm{D}$ printing material. The prototype consists of three segments and two variable structure mechanisms. The 1 st clutch motor and 2 nd clutch motor can control the rotation of cam to regulate seven different locking and release states. The lengths of four cables, denoted as cable $A$, cable $B$, cable $C$, and cable $D$, can be controlled by four DC motor respectively. In this prototype, the original cable length of each segment is 100 $\mathrm{mm}$. The distance of cable holes $d$ is $60 \mathrm{~mm}$.

The control system shown in Figure 10 mainly includes power supply, main controller, motor driver and Bluetooth communication. The power supply part uses a high-power dynamic battery with high output power and large current, which meets the power requirement of multiple motors at the same time. Romeo BLE mini controller equipped with the ATmega328p control chip was adopted as the main controller, which is suitable for the design of desktop micro-robot. The main controller can program four DC motors to retract and release the cables that are symmetrical 90-degree distribution. Two clutch motors also can be controlled to change the state from the seven variable states. Meanwhile, the main

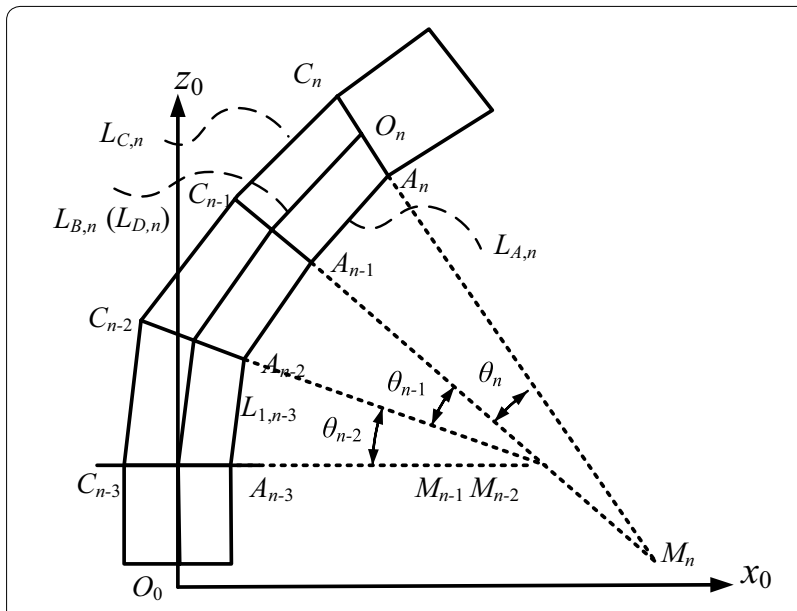

Figure 9 The special case of $N$ segments model in plane

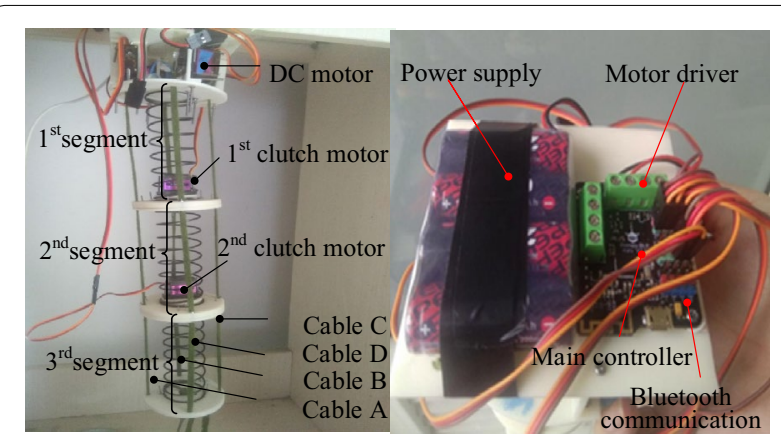

Figure 10 Mechanical model prototype and control system

controller has 8 digital interfaces and 4 analog interfaces, which makes it convenient to connect various sensors for closed-loop control in the future. In addition, the controller integrates a Bluetooth 4.0 communication chip of CC2540, which supports remote control and code update download through the Bluetooth communication. In our experiments, we adopt the open-loop control scheme to control the length of cables.

\subsection{Experiment Results}

According to the mechanical prototype and the simplified control method proposed above, relevant experiments were carried out to analyze the bending and shrinking motion of each segment (Additional file 2).

Figure 11 shows a series of screenshots of continuous deformation toward left direction. In this case, two variable structure mechanisms should control to the state of Figure 11(a). Therefore,the cable $A$ in the first segment and the cable $C$ in the second segment are locked. If the DC motor drives the cable $C$ to contract, the manipulator will bend to the left. Similarly, if two variable structure mechanisms are the state of Figure 11(c). The cable $C$ in the first segment and the cable $C$ in the second segment are locked, the model will be bent to the right direction under the force of the cable $A$. Figure 12 shows a series of screenshots of the continuous deformation. It can be seen that the continuous bending motion of the model can be achieved. Meanwhile,

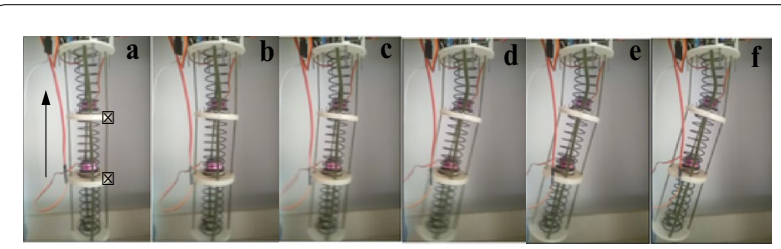

Figure 11 Series screenshots results of left bending 


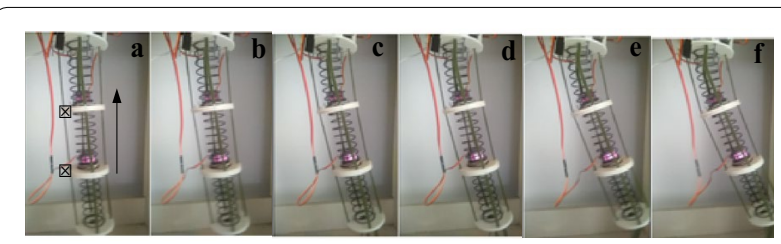

Figure 12 Series screenshots results of right bending

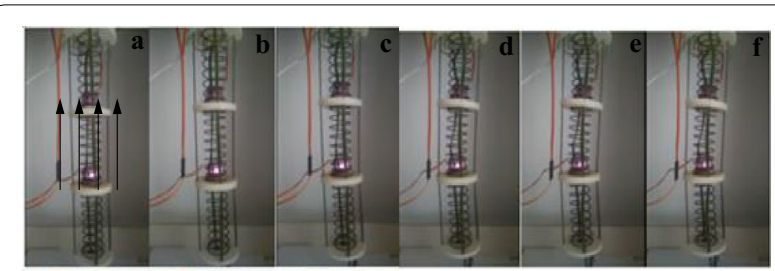

Figure 13 Series screenshots results of shrinking

we can also obtain similar considerable results if we control the prototype to bend to other directions.

If both of two variable structure mechanisms are the state of Figure 5, all four cables are released. The four motors can drive four cables to shrink at the same time, and the model will shrink along the axis. Figure 13 shows the model changes continuously during shrinkage. It can be seen that the function of global shrinking can be realized, but a certain side of bending occurs. The reason for the result is that the shrink in the control of four cables is not synchronized. Meanwhile, different friction exists in the symmetrical structure. The parameter error and the friction can be reduced by using the better performance motor with a robust control algorithm.

The spatial position and deformation of the model are determined by both the states of two variable structure mechanisms and the length of four cables. In order to verify the function of the variable structure mechanism, we made the four cable-driven motors work in the same way and control the rotation angle of cam to change different lock-release states of two variable structure mechanisms Figure 14(a) gives a series of screenshots of the continuous deformation of the first segment when both two variable structure mechanisms are controlled to lock cable $A$ and cable $C$. We found that only the first segment is deformed while the other two segments have no deformation. If only the second variable structure mechanism is controlled to lock cable $A$ and cable $C$ as shown in Figure 14(b), the two segments are deformed even though we give the same cable control. If both two variable mechanisms keep release state, all of the three segments would have motions. Figure 14(c) shows a series of screenshots of the continuous deformation of all the three segments when the cables are contracted.

In order to analysis different spatial positions and variable motion patterns of three cases shown in Figure 14. We supplied the same open-loop control scheme to change the length of cables. In this experiment, the changed length of cable is setting as $60 \mathrm{~mm}$ in all three cases. Because the three cases have different states of variable structure mechanism, different segments have different motion configurations and spatial positions. The experimental results of case 1 can be compared with the corresponding simulation results by the triangle markers described in Figure 15. After meshing a series of screenshots of the continuous deformation by the MATLAB, we can obtain the deformation values and configuration under different locking-release states. The simulation and experiment results of three cases are described in Figure 16. The displace of the end-effector are approximately $(-201,172),(-160$, $204)$, and $(-120,235)$ respectively under the three states above. The offsets and configuration of the models under different variable structure states are different, and the results have a negative correlation. The more controllable the model is, the smaller the curvature of the model is even though the same control of cables is done. This verifies the effectiveness of the proposed model with the variable structure mechanism. The flexible manipulator is capable of achieving continuous deformation.

The experimental results can be compared with the simulation results. The simulation results are described in Figure 15 by the triangle markers. There are about 1-2 $\mathrm{cm}$ errors with the relative error less than $6.8 \%$ between the simulation results and experimental results, through the simple independent control of each segment using the variable structure mechanism. The reasons for causing this error are manifold. The first reason is that there is no sensor feedback and we only adopt the open-loop control. The second reason is that we didn't account for those factors in applications, such as slack cables and vibrating spring.

\section{Conclusions}

In this paper, inspired by the variable motion patterns and muscle of the octopus tentacles, a flexible bio-tensegrity manipulator was proposed to mimic the shrinking behavior and achieve the variable motion patterns of each segment. Some proof-of-concept experiments were conducted to validate the feasibility and validity of our proposed prototype. The following conclusions are drawn: 

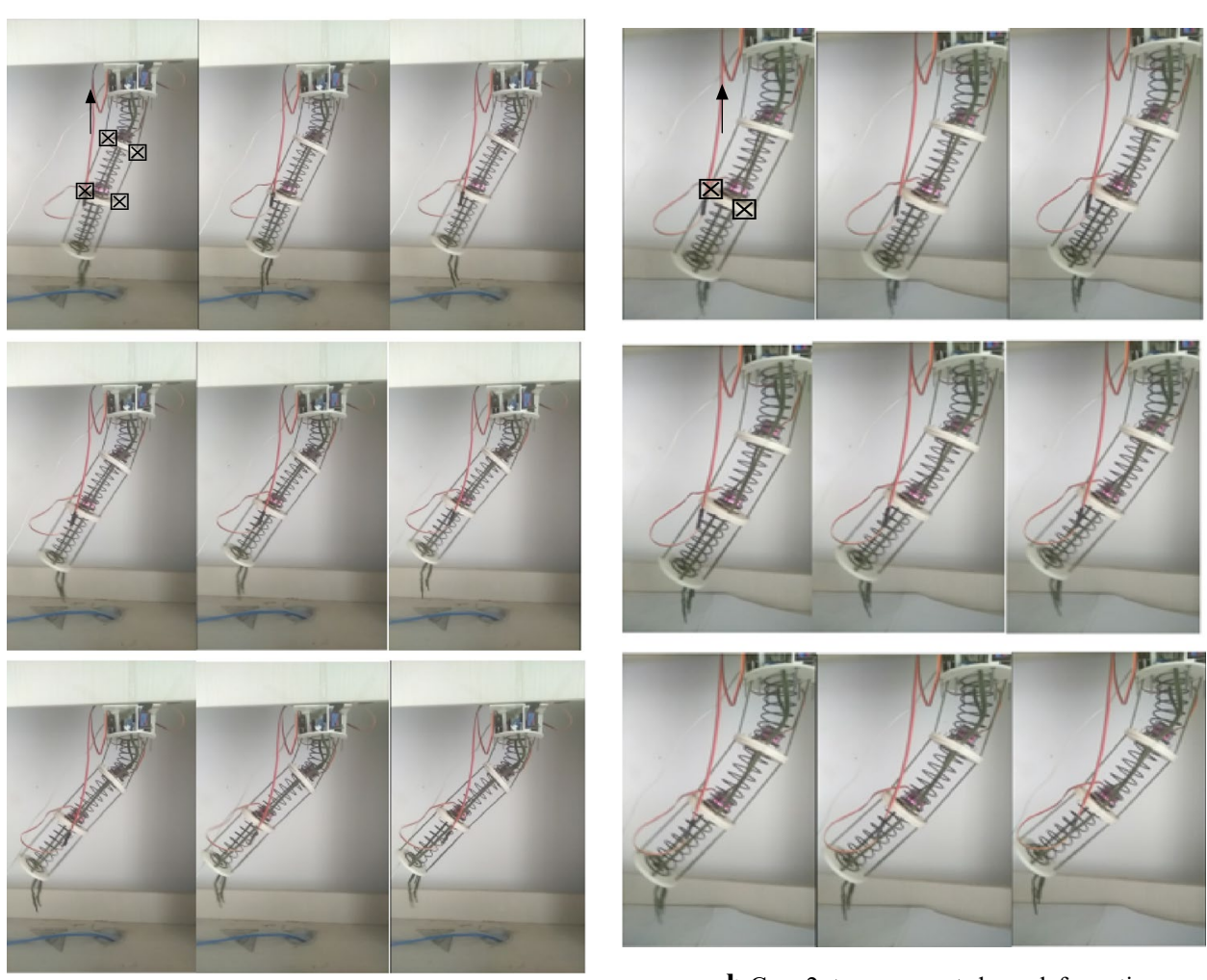

a Case 1: only the first segment has deformation

b Case 2: two segments have deformation
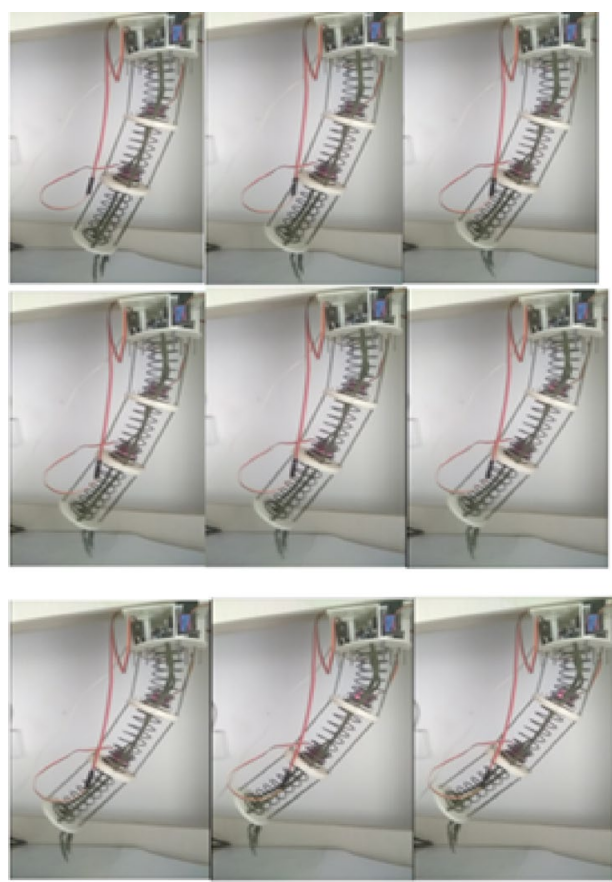

c Case 3: all three segments have deformation

Figure 14 Series screenshots results with different states of variable structure mechanisms 


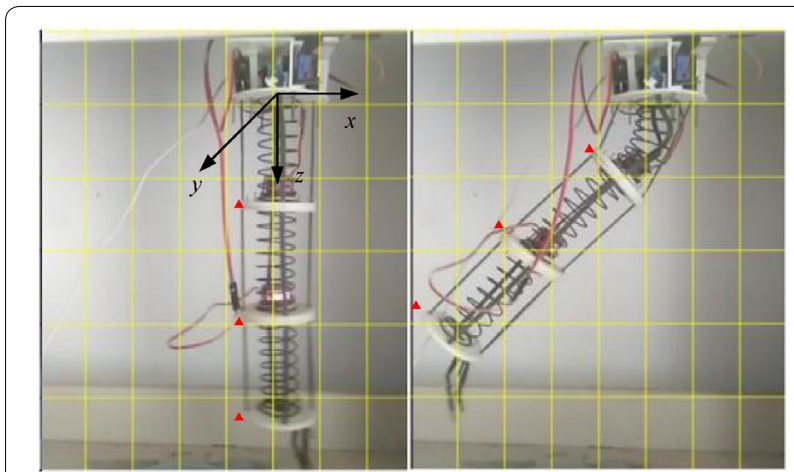

Figure 15 Simulation and experimental results of series screenshots

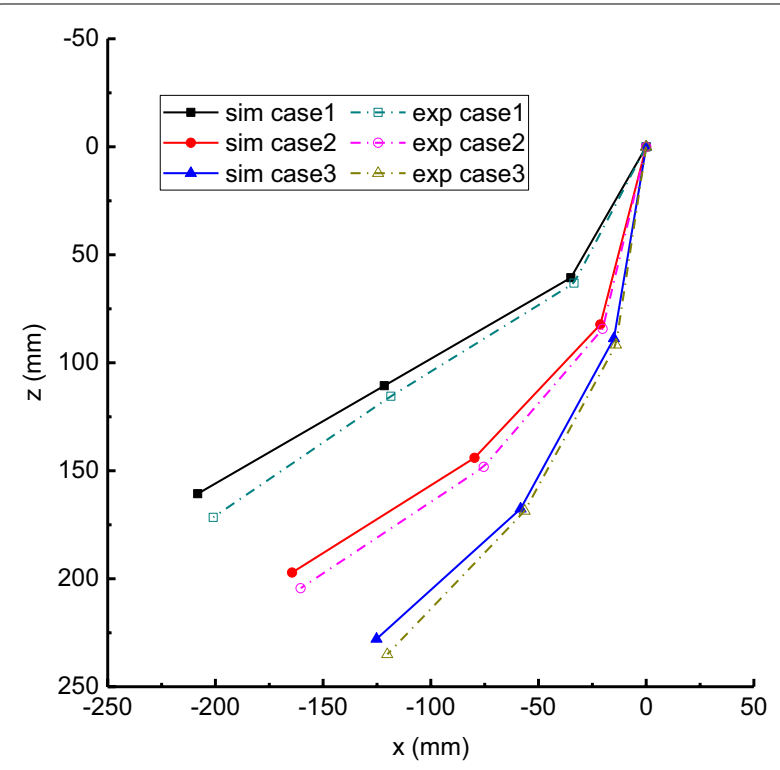

Figure 16 Simulation and experimental results with different states of variable structure mechanism

(1) The flexible bio-tensegrity manipulator combines the elastic element spring and cable-driven to mimic soft structure. Compared with other cable-driven continuum robots, this flexible biotensegrity manipulator not only shows the bending motion, but also can demonstrate the shrinking motion that not featured in the other cable-driven continuum robots. Meanwhile, the proposed manipulator has the characteristics of Multi-DOF and high flexibility, which extended their environmental adaptability and manipulation capability.

(2) The main variable structure mechanism innovatively contains seven kinds of lock-release states to achieve the lock and release of different cables. In this way, we control the length of each cable and adjust the motion patterns of each segment. Compared with other multi-section continuum robots [25], the independent control of each segment is realized under the conditions of without adding more actuation power source.

(3) We then designed and fabricated a mechanical prototype. One open-loop control method was utilized to adjust the lock-release states and control the cable length. By this way, the flexible biotensegrity manipulator can achieve the considerable motion and the variable motion patterns. We also perform some relative experiments, such as bending motion, shrinking motion, and variable mechanisms motion, for demonstrating its high flexibility, environmental adaptability, manipulation capability.

In the future, we need to integrate the system more tightly with sensor devices and considerable control scheme to fully demonstrate its abilities. We are also considering to perform closed-loop feedback control to realize more precise position and stiffness control. Meanwhile, those influence factors in applications which are not analyzed here, such as slack cables, transmission clearance, and vibrating spring, should be taken into account. Moreover, the range of its speed and stiffness within its workspace should be carefully evaluated and directly compared with other existing research results. In order to improve reliability and expand its application area, modular design can be adopted. If each module can be conveniently arranged in series and replaced, the reliability of the whole manipulator will be greatly improved, and this modularization is easy to be popularized.

\section{Supplementary information}

Supplementary information accompanies this paper at https://doi. org/10.1186/s10033-019-0426-7.

Additional file 1. Octopus R1.

Additional file 2. Experiment R1.

\section{Acknowledgements}

Not applicable.

\section{Authors' Contributions}

DW was in charge of the whole trial; DW, TG, XM, and RX wrote the manuscript; XM and CZ assisted with sampling and laboratory analyses. All authors read and approved the final manuscript.

\section{Authors' Information}

Dunwen Wei, born in 1986, is currently an assistant professor at School of Mechanical and Electrical Engineering, University of Electronic Science and Technology of China, China. His research interests include bioinspired robots, medical and rehabilitation robotics.

Tao Gao, born in 1979, is currently a professor at School of Mechanical and Electrical Engineering, University of Electronic Science and Technology of China, China. His research interests include bioinspired robots. 
Xiaojuan Mo, born in 1992, is currently a PhD candidate at School of Mechanical Engineering, Northwestern Polytechnical University, China. Her research interests include animals' locomotion and bioinspired robots.

Ruru Xi, born in 1985, is currently an assistant professor at School of Mechanical Engineering, Hangzhou Dianzi University, China. Her research interests include dynamic analysis and control of the robotics system.

Cong Zhou, born in 1992, is currently a master at School of Mechanical and Electrical Engineering, University of Electronic Science and Technology of China. His research interests include mechatronics engineering, robot control system.

\section{Funding}

Supported by National Natural Science Foundation of China (Grant Nos. 51705066, 51805128), Sichuan Science and Technology Program (Grant No. 2019YFG0343), and Fundamental Research Funds for the Central Universities of China (Grant Nos. ZYGX2019J041, ZYGX2016KYQD137).

\section{Competing Interests}

The authors declare that they have no competing interests.

\section{Author Details}

1 School of Mechanical and Electrical Engineering, University of Electronic Science and Technology of China, Chengdu 611731, China. ${ }^{2}$ School of Mechanical Engineering, Northwestern Polytechnical University, Xi'an 710072, China. ${ }^{3}$ School of Mechanical Engineering, Hangzhou Dianzi University, Hangzhou 310018, China.

Received: 20 July 2019 Revised: 12 November 2019 Accepted: 27 December 2019

Published online: 03 February 2020

\section{References}

[1] G M Whitesides. Soft robotics. Angewandte Chemie International Edition, 2018, 57(16): 4258-4273.

[2] M C Yip, D B Camarillo. Model-less feedback control of continuum manipulators in constrained environments. IEEE Transactions on Robotics, 2014, 30(4): 880-889.

[3] Y Liu, Z Ge, S Yang, et al. Elephant's trunk robot: An extremely versatile under-actuated continuum robot driven by a single motor. Journal of Mechanisms and Robotics, 2019, 11(5): 051008

[4] M Calisti, G Picardi, C Laschi. Fundamentals of soft robot locomotion. Journal of The Royal Society Interface, 2017, 14(130): 20170101.

[5] R Pfeifer, F lida, M Lungarella. Cognition from the bottom up: On biological inspiration, body morphology, and soft materials. Trends in Cognitive Sciences, 2014, 18(8): 404-413.

[6] C Laschi, B Mazzolai, M Cianchetti. Soft robotics: Technologies and systems pushing the boundaries of robot abilities. Science Robotics, 2016, 1(1): eaah3690.

[7] V Chalvet, D J Braun. Criterion for the design of low-power variable stiffness mechanisms. IEEE Transactions on Robotics, 2017, 33(4): 1002-1010.

[8] D Rus, M TTolley. Design, fabrication and control of soft robots. Nature, 2015, 521(7553): 467-475.

[9] L Hines, K Petersen, G Z Lum, et al. Soft actuators for small-Scale robotics. Advanced Materials, 2017, 29(13): 1603483.

[10] A Zolfagharian, A Z Kouzani, S Y Khoo, et al. Evolution of 3D printed soft actuators. Sensors and Actuators A: Physical, 2016, 250: 258-272.

[11] R L Truby, M Wehner, A K Grosskopf, et al. Soft somatosensitive actuators via embedded 3D printing. Advanced Materials, 2018, 30(15): 1706383.

[12] A Firouzeh, M Salerno, J Paik. Soft pneumatic actuator with adjustable stiffness layers for multi-Dof actuation. 2015 IEEE/RSJ International Conference on Intelligent Robots and Systems (IROS), 2015: 1117-1124.
[13] M A Robertson, H Sadeghi, J M Florez, et al. Soft pneumatic actuator fascicles for high force and reliability. Soft Robotics, 2016, 4(1): 23-32.

[14] A D Marchese, R K Katzschmann, D Rus. A recipe for soft fluidic elastomer robots. Soft Robot, 2015, 2(1): 7-25.

[15] A Shiva, A Stilli, Y Noh, et al. Tendon-based stiffening for a pneumatically actuated soft manipulator. IEEE Robotics and Automation Letters, 2016, 1(2): 632-637.

[16] Z Xu, C Ding, D Wei, et al. Electro and light-active actuators based on reversible shape-memory polymer composites with segregated conductive networks. ACS Applied Materials \& Interfaces, 2019, 11(33): 30332-30340

[17] J E Shim, Y J Quan, W Wang, et al. A smart soft actuator using a single shape memory alloy for twisting actuation. Smart Materials and Structures, 2015, 24(12): 10.

[18] H Yang, M Xu, W Li, et al. Design and implementation of a soft robotic arm driven by sma coils. IEEE Transactions on Industrial Electronics, 2018, 66(8): 6108-6116.

[19] Z Zhakypov, K Mori, K Hosoda, et al. Designing minimal and scalable insect-inspired multi-locomotion millirobots. Nature, 2019, 571(7765): 381-386.

[20] H Wang, J Chen, HY Lau, et al. Motion planning based on learning from demonstration for multiple-segment flexible soft robots actuated by electroactive polymers. IEEE Robotics and Automation Letters, 2016, 1(1): 391-398.

[21] R Mutlu, G Alici, W Li. A soft mechatronic microstage mechanism based on electroactive polymer actuators. IEEE/ASME Transactions on Mechatronics, 2015, 21(3): 1467-1478.

[22] J Zhang, J Sheng, CT O'neill, et al. Robotic artificial muscles: Current progress and future perspectives. IEEE Transactions on Robotics, 2019, 35(3): 761-781.

[23] H Wang, C Wang, W Chen, et al. Three-dimensional dynamics for cabledriven soft manipulator. IEEE/ASME Transactions on Mechatronics, 2016, 22(1): 18-28.

[24] F Renda, F Boyer, J Dias, et al. Discrete cosserat approach for multisection soft manipulator dynamics. IEEE Transactions on Robotics, 2018, 34(6): 1518-1533.

[25] I S Godage, R J Webster, I D Walker. Center-of-gravity-based approach for modeling dynamics of multisection continuum arms. IEEE Transactions on Robotics, 2019, 35(5): 1097-1108.

[26] A Yeshmukhametov, K Koganezawa, Y Yamamoto. Design and kinematics of cable-driven continuum robot arm with universal joint backbone. 2018 IEEE International Conference on Robotics and Biomimetics (ROBIO), 2018: 2444-2449.

[27] G A Pratt, M M Williamson. Series elastic actuators. Proceedings 1995 IEEE/ RSJ International Conference on Intelligent Robots and Systems. Human Robot Interaction and Cooperative Robots, 1995: 399-406.

[28] R V Ham, T Sugar, B Vanderborght, et al. Compliant actuator designs. IEEE Robotics \& Automation Magazine, 2009, 3(16): 81-94.

[29] D Wei, W Ge. Fuzzy coordinated control of contact constraint problem for robot system with compliant actuators. Proceedings of the Institution of Mechanical Engineers, Part I: Journal of Systems and Control Engineering, 2016, 230(7): 640-650

[30] J Cai, X Wang, X Deng, et al. Form-finding method for multi-mode tensegrity structures using extended force density method by grouping elements. Composite Structures, 2018, 187: 1-9.

[31] Z G Joey, A A Calderón, L Chang, et al. An earthworm-inspired frictioncontrolled soft robot capable of bidirectional locomotion. Bioinspiration \& biomimetics, 2019, 14(3): 036004

[32] Y Cao, Y Liu, Y Chen, et al. A novel slithering locomotion mechanism for a snake-like soft robot. Journal of the Mechanics and Physics of Solids, 2017 99: 304-320.

[33] C Laschi, B Mazzolai, V Mattoli, et al. Design of a biomimetic robotic octopus arm. Bioinspiration \& Biomimetics, 2009, 4(1): 015006. 